\title{
Bronchial smooth muscle remodeling involves calcium-dependent enhanced mitochondrial biogenesis in asthma
}

\author{
Thomas Trian, ${ }^{1,2}$ Giovanni Benard, ${ }^{3}$ Hugues Begueret, $, 2,4$ \\ Rodrigue Rossignol, ${ }^{3}$ Pierre-Olivier Girodet, ${ }^{1,2,4}$ Debajyoti Ghosh, ${ }^{1,2}$ \\ Olga Ousova, ${ }^{1,2}$ Jean-Marc Vernejoux, ${ }^{4}$ Roger Marthan, ${ }^{1,2,4}$ \\ José-Manuel Tunon-de-Lara, ${ }^{1,2,4}$ and Patrick Berger ${ }^{1,2,4}$
}

1Universite Bordeaux 2, Laboratoire de Physiologie Cellulaire Respiratoire, F-33076 Bordeaux, France

Institut National de la Santé et de la Recherche Médicale (INSERM), ${ }^{2}$ U885 and ${ }^{3}$ U688, F-33076 Bordeaux, France

${ }^{4}$ Centre Hospitalier Universitaire de Bordeaux, F-33076 Bordeaux, France

Asthma and chronic obstructive pulmonary disease (COPD) are characterized by different patterns of airway remodeling, which all include an increased mass of bronchial smooth muscle (BSM). A remaining major question concerns the mechanisms underlying such a remodeling of BSM. Because mitochondria play a major role in both cell proliferation and apoptosis, we hypothesized that mitochondrial activation in BSM could play a role in this remodeling. We describe that both the mitochondrial mass and oxygen consumption were higher in the BSM from asthmatic subjects than in that from both COPD and controls. This feature, which is specific to asthma, was related to an enhanced mitochondrial biogenesis through up-regulation of peroxisome proliferator-activated receptor $\gamma$ coactivator (PGC)$1 \alpha$, nuclear respiratory factor-1, and mitochondrial transcription factor $A$. The priming event of such activation was an alteration in BSM calcium homeostasis. BSM cell apoptosis was not different in the three groups of subjects. Asthmatic BSM was, however, characterized by increased cell growth and proliferation. Both characteristics were completely abrogated in mitochondria-deficient asthmatic BSM cells. Conversely, in both COPD and control BSM cells, induction of mitochondrial biogenesis reproduced these characteristics. Thus, BSM in asthmatic patients is characterized by an altered calcium homeostasis that increases mitochondrial biogenesis, which, in turn, enhances cell proliferation, leading to airway remodeling.

\section{CORRESPONDENCE}

Patrick Berger:

patrick.berger@u-bordeaux2.fr

Abbreviations used: ANOVA, analysis of variance; BSM, bronchial smooth muscle; CaMK-IV, calcium/calmodulindependent protein kinase IV; COPD, chronic obstructive pulmonary disease; mtTFA, mitochondrial transcription factor A; NRF, nuclear respiratory factor; PGC, peroxisome proliferator-activated receptor $\gamma$ coactivator.
Asthma and chronic obstructive pulmonary disease (COPD) are inflammatory airway diseases that are characterized by different patterns of airway remodeling (1). Nevertheless, the decrease in lung function that characterizes both diseases is associated with an increased mass of bronchial smooth muscle (BSM) (2, 3), which is likely to be the most important abnormality responsible for the airway narrowing observed in response to bronchoconstricting stimuli (4). The mechanisms underlying such remodeling of smooth muscle remain largely unknown. On the one hand, in smooth muscle from asthmatic patients, excessive in vitro proliferation of BSM cells has been demonstrated (5-7). In addition, decreased apoptosis of BSM cells has also been demon-

The online version of this article contains supplemental material. strated, although this was in a rat model of experimental asthma (7). On the other hand, in COPD, smooth muscle remodeling appears limited to airways distal to the fourth generation $(3,8)$. Although an increase in TGF- $\beta 1$ production by BSM cells has been proposed, such localization and the complete mechanism remain unexplained (9). Whatever its cause, BSM remodeling is poorly sensitive to current therapeutics in both asthma and COPD.

Mitochondria play a major role in both cell proliferation and apoptosis $(10,11)$. In cancer, for instance, targeting of mitochondrial function and mitochondrial antiapoptotic protein bcl-2 has been used to either suppress the proliferation of tumor cells (10) or induce cell apoptosis in solid tumors (12). Mitochondria are also involved in other diseases, such as neuron-degenerative 
diseases (11). However, their role in asthma or COPD remains to be investigated. We hypothesized that mitochondrial activation in BSM from asthmatic or COPD patients could contribute to smooth muscle remodeling. To investigate this hypothesis, we have compared mitochondrial mass, activity, and biogenesis in BSM obtained from asthmatics, COPD patients, and normal controls. We describe that both the mitochondrial mass and oxygen consumption were higher in the BSM from asthmatic subjects than in that from both COPD and controls. This feature, which is specific to asthma, is related to an enhanced mitochondrial biogenesis as a consequence of an increase in extracellular calcium influx upon activation of asthmatic BSM cells. We also demonstrate a specific mitochondria-dependent pathway for asthmatic BSM cell proliferation. Targeting such a pathway may thus represent a new approach for the treatment of airway remodeling in asthma.

\section{RESULTS}

\section{Clinical populations}

The clinical characteristics of all subjects are shown in Table I. All of the 14 severe persistent asthmatics were lifelong nonsmokers and received stable treatments, including oral or inhaled corticosteroids and $\beta 2$ agonists. 10 of them were atopic. The 17 moderate to severe COPD patients were either current or former smokers, and 9 of them received stable treatments, including oral or inhaled corticosteroids and/or $\beta 2$ agonists. None of the asthmatic or COPD patients experienced a recent $(<3 \mathrm{mo})$ exacerbation of the disease. The mean duration of the disease in asthmatic and COPD patients was $26 \pm 4.6$
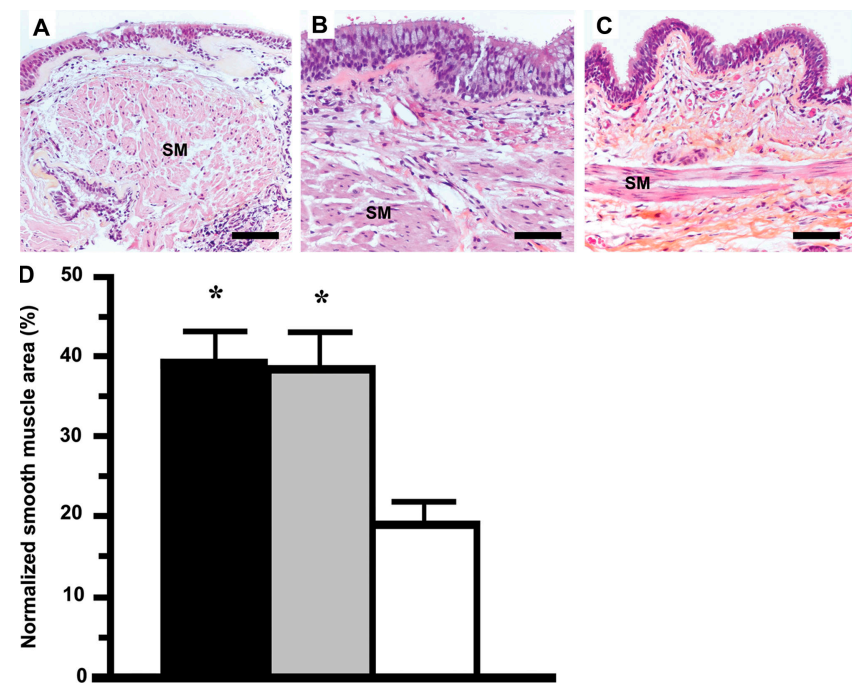

Figure 1. BSM remodeling in both asthma and COPD. Representative optic microscopic images from bronchial sections stained with HES were obtained from an asthmatic (A), a COPD (B), or a control subject (C) and observed at 200x magnification. Smooth muscles were visualized (SM). Bars, $50 \mu \mathrm{m}$. (D) The normalized smooth muscle area was assessed from microscopic images. Bronchial specimens were obtained from asthmatic (black column; $n=10$ ), COPD (gray column; $n=7$ ), and control subjects (white column; $n=6$ ). Data are the mean \pm the SEM. ${ }^{,}, \mathrm{P}<0.05$ between populations using ANOVA with the use of Bonferroni's test.

and $18 \pm 2.8 \mathrm{yr}$, respectively. Of the 19 control subjects who received no treatment, 8 of them were lifelong nonsmokers, whereas 11 were former smokers.

Table I. Clinical and functional characteristics of subjects

\begin{tabular}{|c|c|c|c|}
\hline Characteristic & Patients with asthma & Patients with COPD & Controls \\
\hline No. of patients & 14 & 17 & 19 \\
\hline Age (yr) & $42.3 \pm 5.7$ & $59.8 \pm 3.0$ & $60.4 \pm 2.5$ \\
\hline BMI $\left(\mathrm{kg} / \mathrm{m}^{2}\right)$ & $26.9 \pm 1.1$ & $24.0 \pm 0.9$ & $27.4 \pm 1.1$ \\
\hline \multicolumn{4}{|l|}{ Smoking history } \\
\hline Pack years & $0 \pm 0$ & $46.3 \pm 4.8$ & $19.5 \pm 5.7$ \\
\hline Current (no. of patients) & 0 & 8 & 0 \\
\hline Former (no. of patients) & 0 & 9 & 11 \\
\hline Years since quitting & - & $11.4 \pm 4.8$ & $13.1 \pm 3.8$ \\
\hline \multicolumn{4}{|l|}{ Treatments } \\
\hline LABA (no. of patients) & 11 & 6 & 0 \\
\hline ICS (no. of patients) & 10 & 5 & 0 \\
\hline OCS (no. of patients) & 6 & 3 & 0 \\
\hline \multicolumn{4}{|l|}{$\mathrm{FEV}_{1}$} \\
\hline Liters & $2.2 \pm 0.2$ & $1.8 \pm 0.1$ & $2.9 \pm 0.1$ \\
\hline Percentage of predicted value & $83.1 \pm 6.3$ & $60.2 \pm 2.5$ & $101.7 \pm 3.2$ \\
\hline $\mathrm{FEV}_{1}: \mathrm{FVC}$ ratio (\% of FVC) & $71.9 \pm 3.7$ & $56.3 \pm 2.2$ & $81.8 \pm 1.7$ \\
\hline \multicolumn{4}{|l|}{ FEF 25-75 } \\
\hline Liters $\sec ^{-1}$ & $1.9 \pm 0.3$ & $0.9 \pm 0.1$ & $2.9 \pm 0.1$ \\
\hline Percentage of predicted value & $54.6 \pm 7.8$ & $28.6 \pm 3.1$ & $90.2 \pm 4.0$ \\
\hline
\end{tabular}

Data are the mean \pm the SEM. BMI, body mass index; LABA, long-acting $\beta 2$ agonist; ICS, inhaled corticosteroid; OCS, oral corticosteroid; FEV $_{1}$, forced expiratory volume in one second; FVC, forced vital capacity; FEF 25-75, forced expiratory flow between 25 and $75 \%$ of FVC. 
Mitochondrial mass and activity are increased only in the BSM of asthmatics

A morphological analysis of BSM was performed in the three groups of subjects (Fig. 1, A-C). BSM mass was increased in both asthmatic and COPD patients as compared with controls (Fig. $1 \mathrm{D} ; \mathrm{P}=0.01)$. Ultrastructure of BSM mitochondria was then compared in asthma and COPD to control subjects (Fig. 2, A-C). The number of mitochondrial sections was higher in the BSM of asthmatics than in that of both COPD and controls (Fig. $2 \mathrm{D} ; \mathrm{P}<0.001)$. The mean area per section was, however, unchanged $(0.1 \pm 0.007,0.1 \pm 0.016$, and $0.1 \pm$ $0.009 \mu \mathrm{m}^{2}$ for asthmatics, COPD, and controls, respectively; analysis of variance [ANOVA] $\mathrm{P}=0.87$ ). Collectively, these results favor the hypothesis that the increase in mitochondrial mass in the BSM of asthmatics is related to an increase in number rather than in individual size. Similar results were obtained with cultured growth-arrested BSM cells (Fig. S1, A-D, available at http://www.jem.org/cgi/content/full/jem
.20070956/DC1; $\mathrm{P}=0.02)$. There was a significant increase in the mitochondrial density in asthmatic BSM cells both ex vivo (Fig. $2 \mathrm{E} ; \mathrm{P}=0.01$ ) and in vitro (Fig. $\mathrm{S} 1 \mathrm{E} ; \mathrm{P}=0.01$ ) as compared with both COPD and controls. Based on electronic microscopy, the mitochondrial density has been shown to reflect the mitochondrial activity $(13,14)$. To assess the smooth muscle specificity of these results, we also analyzed ultrastructural mitochondrial parameters in other cell types from the same bronchial specimens. There was no difference between the three groups, in terms of both number $(\mathrm{P}=0.35$ and $\mathrm{P}=0.89)$ and density of mitochondria $(\mathrm{P}=0.47$ and $\mathrm{P}=$ 0.87 ) in endothelial and epithelial cells, respectively (unpublished data). The increased mass of asthmatic BSM mitochondria was further confirmed, in vitro, by an increase in the porin content compared with that of both COPD and controls (Fig. $2 \mathrm{~F} ; \mathrm{P}=0.001$ ). We also found within the asthmatic population that both duration of the disease and forced expiratory volume in one second $\left(\mathrm{FEV}_{1}\right) /$ forced vital capacity
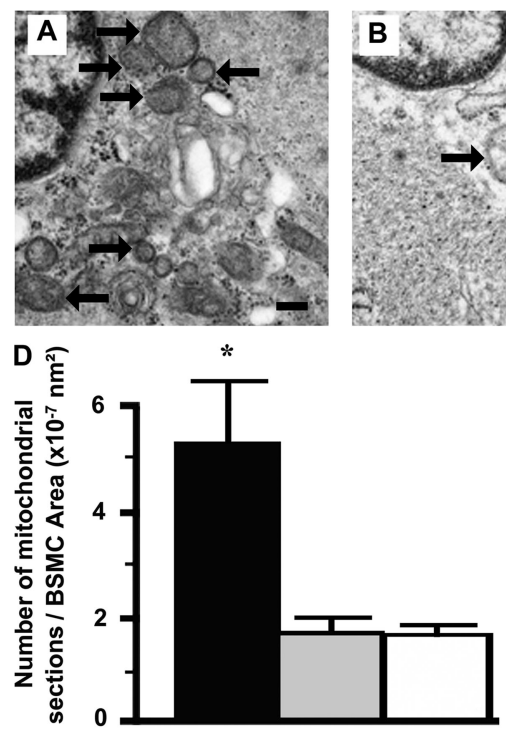

$\mathbf{F}$
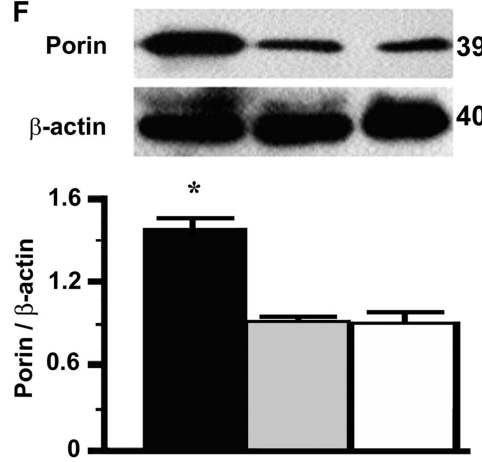

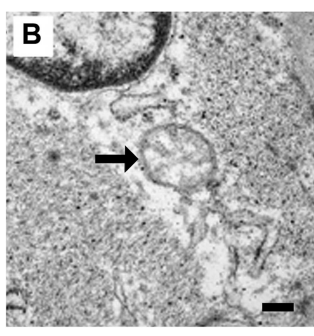

E

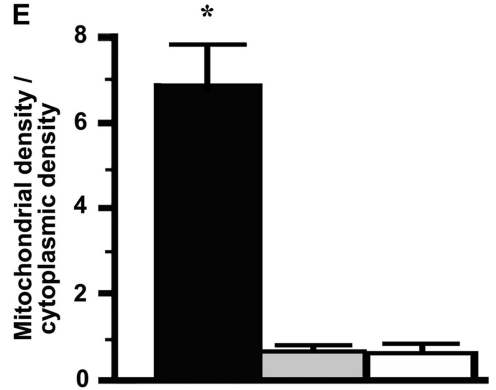

G
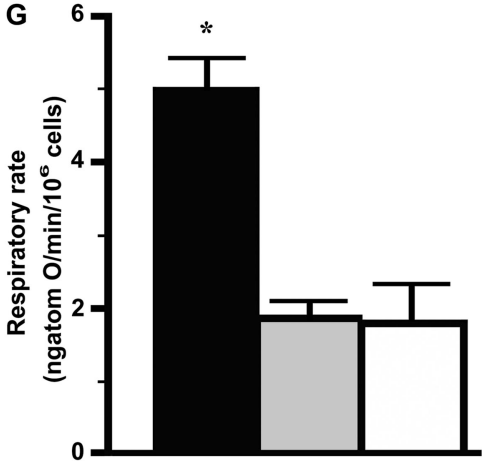

Figure 2. Increased mitochondrial mass and activity in asthmatic BSM. Representative electronic microscopic images from bronchial sections were obtained from an asthmatic (A), a COPD (B), or a control subject (C) and observed at 26,000x magnification. Some smooth muscle mitochondria were visualized (arrows). Bars, $0.2 \mu \mathrm{m}$. The number (D) and the density (E) of mitochondria were assessed from electronic microscopic images $(n=4$ for each population). Mitochondrial mass was assessed by the porin content using Western blot (F; $n=8$ for asthmatics, $n=5$ for $\operatorname{COPD}$, and $n=7$ for controls). Endogenous cellular oxygen consumption was evaluated by oxygraphy (G; $n=5$ for asthmatics, $n=4$ for COPD, and $n=4$ for controls). BSM cells (BSMC) were obtained from asthmatic (black columns), COPD (gray columns), and control subjects (white columns). Data are the mean \pm the SEM. ${ }^{*}, \mathrm{P}<0.05$ between populations using ANOVA with the use of Bonferroni's test. 
(FVC) ratio were correlated with the porin content $(\mathrm{r}=0.77$, $\mathrm{P}=0.03$ and $\mathrm{r}=-0.84, \mathrm{P}=0.01$, respectively), whereas no correlation was found within the COPD or the control population. Similarly, the oxygen consumption of asthmatic BSM cells was specifically enhanced compared with COPD or control BSM cells (Fig. $2 \mathrm{G}$; P = 0.01). Such an increase in the mitochondrial respiratory rate was not associated with differences in the coupling degree, as demonstrated by the effects of inhibitors of the phosphorylation system. Similarly, oxygen consumption of COPD and control BSM, although lower, also reflected coupled mitochondrial respiration (Fig. S2; P < 0.04 , paired $t$ tests). These inhibitors decreased the mitochondrial respiration of asthmatics, COPD, and controls by 18.6, 21.2, and $24.7 \%$, respectively, whereas cyanide completely inhibited oxygen consumption of all BSM cells. The increased mitochondrial respiration in asthma thus appears to result from the increased organelle content and subsequent enhancement in the overall oxidative capacity in BSM.

\section{Mitochondrial biogenesis is increased in asthmatic BSM cells through a calcium-dependent pathway}

We next analyzed the mitochondrial network by confocal microscopy (Fig. 3, A-C). Surprisingly, asthmatic BSM cells presented a typical aspect of intense mitochondrial biogenesis, as shown by the presence of several budding areas with intense dots, and a wider network with increased ramification (Fig. $3 \mathrm{~A}$ ). Because the mitochondrial transcription factor A (mtTFA) is the main factor involved in mitochondrial biogenesis, we measured its protein content and transcription level in the three groups of BSM. As compared with both controls and COPD, mtTFA was increased in asthmatic BSM cells (Fig. 3,
D and E). Upstream mechanisms that would explain such an increased mitochondrial biogenesis were then examined. The two transcription factors nuclear respiratory factor-1 (NRF-1) and peroxisome proliferator-activated receptor $\gamma$ coactivator$1 \alpha(\mathrm{PGC}-1 \alpha)$ were both elevated in asthmatic BSM cells at both the protein and the transcription levels (Fig. 3, D and E). Among the various factors that could activate PGC- $1 \alpha$, we observed that the calcium/calmodulin-dependent protein kinase IV (CaMK-IV) was phosphorylated and thus activated in asthmatic BSM cells compared with controls and COPD (Fig. 4 A). We thus investigated whether calcium homeostasis was deregulated in asthmatic BSM cells using microspectrofluorimetry. The resting calcium concentration was consistent in asthmatic $(125 \pm 8 \mathrm{nM})$, COPD $(127 \pm 7 \mathrm{nM})$, or control BSM cells $(133 \pm 4 \mathrm{nM} ; \mathrm{P}=0.60)$. However, the calcium response to acetylcholine was significantly altered in asthmatic BSM cells (Fig. 4, B-D). Whereas the amplitude of the calcium rise was unchanged $(275 \pm 20,284 \pm 43$, and $272 \pm 27 \mathrm{nM}$ in asthmatics, COPD, and controls, respectively; $\mathrm{P}=0.95)$, the area under the curve was significantly increased in asthmatic BSM cells (Fig. 4 E). A similar result was obtained in asthmatic BSM cells stimulated with histamine, confirming that it was not agonist-specific (Fig. S3, A-D, available at http://www .jem.org/cgi/content/full/jem.20070956/DC1). An enhanced calcium influx in asthmatic BSM cells accounts for this result, as both removal of extracellular calcium using EGTA and blockade of calcium channels using methoxyverapamil (D600) abolished such abnormal calcium responses in asthmatic BSM cells (Fig. 4 E and Fig. S3 D). Blockade of calcium influx using D600 also inhibited the activation of CaMK-IV (Fig. 4 A) and the subsequent activation of mitochondrial biogenesis through
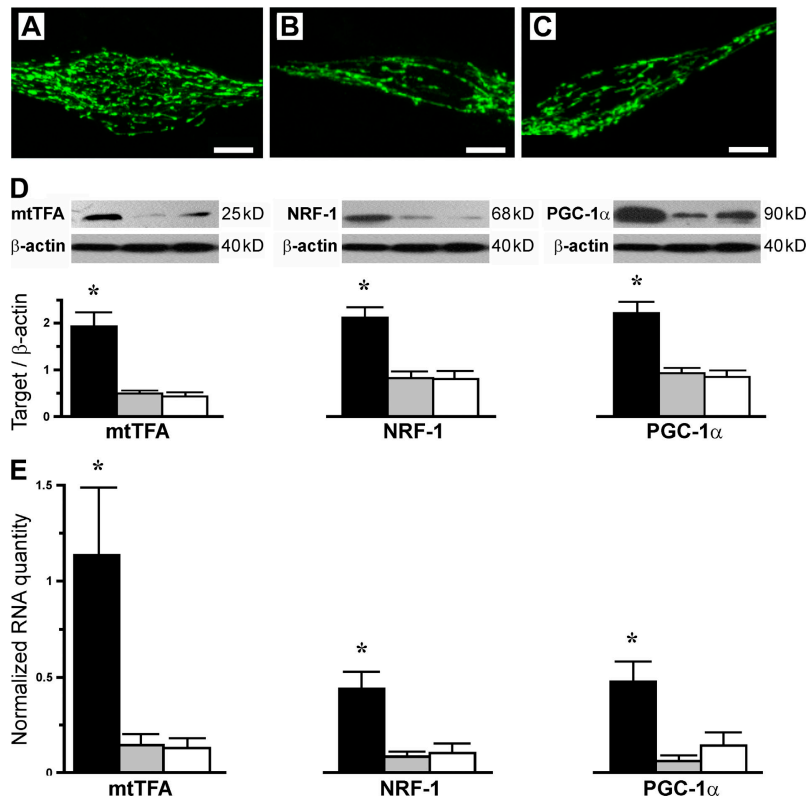

Figure 3. Increased mitochondrial biogenesis in asthmatic BSM. Representative confocal images of the mitochondrial network after three dimensional reconstruction were obtained from asthmatic (A), COPD (B), or control (C) BSM cells. Bars, $10 \mu \mathrm{m}$. mtTFA, NRF-1, and PGC-1 $\alpha$ levels were assessed by both Western blot (D) and quantitative RT-PCR (E). BSM cells were obtained from asthmatic (black columns; $n=6$ ), COPD (gray columns; $n=7$ ), and control subjects (white columns; $n=6$ ). Data are the mean \pm the SEM. ${ }^{*}, \mathrm{P}<0.05$ between populations using ANOVA with the use of Bonferroni's test. 
PGC-1 $\alpha$, NRF-1, and mtTFA (Fig. 5, A-C), leading to the increase in the mitochondrial mass as assessed by the porin content (Fig. 5 D). Thus, these results indicate that an enhanced extracellular calcium influx, specific to asthmatic BSM cells, is the initial priming event leading to an increased mitochondrial biogenesis and mass.

\section{Only asthmatic BSM cell proliferation is mitochondria dependent}

To further assess the specific role of mitochondria in asthmatic BSM proliferation contributing to airway remodeling, we next compared BSM cell growth in the presence or absence

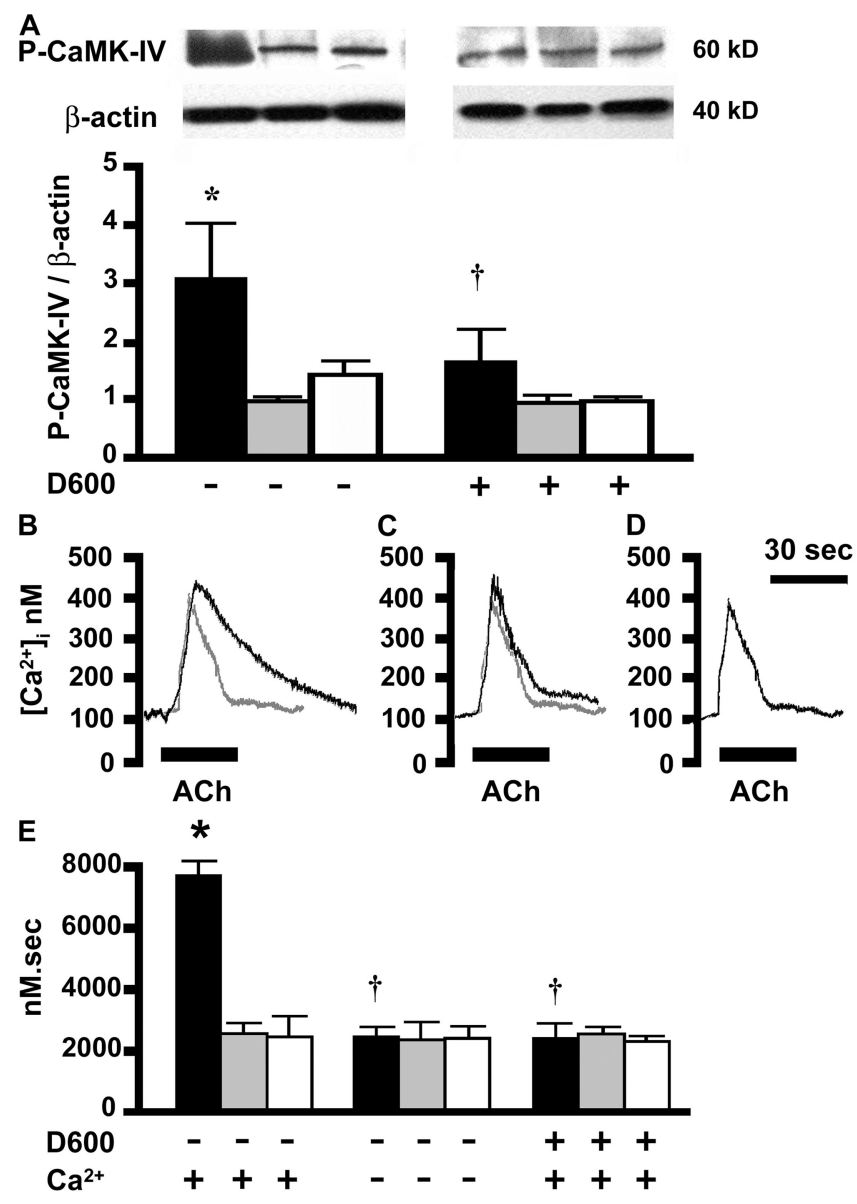

Figure 4. Altered cell calcium homeostasis in asthmatic BSM. (A) Phosphorylated CaMK-IV (P-CaMK-IV) levels were assessed by Western blot. Representative intracellular calcium responses after stimulation for $30 \mathrm{~s}$ by $10^{-5} \mathrm{M}$ acetylcholine (ACh) are presented in BSM cells from asthmatic (B), COPD (C), or control subjects (D). As a reference, response from the control cell (D) is presented as a gray line ( $B$ and $C)$. The area under the curve was assessed from the calcium response (E). BSM cells were analyzed in the absence (-) or presence (+) of $2 \mathrm{mM}$ extracellular $\mathrm{Ca}^{2}+$ or $1 \mu \mathrm{M}$ methoxyverapamil (D600). Cells were obtained from asthmatic (black columns; $n=5$ ), COPD (gray columns; $n=4$ ), and control subjects (white columns; $n=4$ ). Data are the mean \pm the SEM. ${ }^{*}, \mathrm{P}<0.05$ between populations within an experimental condition using ANOVA with the use of Bonferroni's test.,$+ \mathrm{P}<0.05$ between experimental conditions versus 2 $\mathrm{mM} \mathrm{Ca}{ }^{2+}$ without $\mathrm{D} 600$ within a population using paired Student's $t$ tests. of anaerobic glycolysis. For this purpose, BSM cell proliferation curves were plotted in the three groups of subjects using either glucose or galactose in the culture medium (Fig. 6, A and B). In the presence of glucose, which allows ATP to be produced by both aerobic and anaerobic glycolysis, asthmatic BSM cell growth was significantly increased compared with that of COPD and control subjects (Fig. $6 \mathrm{~A} ; \mathrm{P}=0.02$ ), with a concomitant decrease in the doubling time (Fig. $6 \mathrm{C}$; $\mathrm{P}=$ 0.02). When galactose, which only allows cells to produce ATP by mitochondrial oxidative phosphorylations, was substituted for glucose, the doubling time of the cell growth from both COPD $(P=0.02)$ and control subjects $(P=0.02)$ significantly increased, whereas that of asthmatic cells remained constant (Fig. 6, B and C). Because an increase in cell growth can be related to a decreased apoptosis and/or an increased proliferation, we analyzed Annexin $\mathrm{V}$ binding and BrdU incorporation, respectively. Taking into account the percentage of Annexin V-positive cells, spontaneous apoptosis was not altered in asthmatic BSM cells as compared with that of COPD and controls (Fig. S4, available at http://www.jem .org/cgi/content/full/jem.20070956/DC1). However, BrdU incorporation increased in BSM cells from asthmatics as compared with that in both COPD and controls in the presence of glucose (Fig. $6 \mathrm{D} ; \mathrm{P}=0.01$ ). Incubation for $11 \mathrm{~d}$ in the absence of glucose significantly inhibited BrdU incorporation in BSM cells from controls $(\mathrm{P}=0.02)$ and COPD patients $(\mathrm{P}=0.02)$, but not in that from asthmatics (Fig. $6 \mathrm{D} ; \mathrm{P}=0.99)$. Collectively, these results demonstrate that the proliferation of asthmatic BSM cells mainly uses mitochondrial-dependent



Figure 5. Effect of methoxyverapamil (D600) on mitochondrial biogenesis and content. PGC-1 $\alpha$ (A), NRF-1 (B), mtTFA (C), and porin (D) levels were assessed by Western blot in BSM cells cultured in the absence $(-)$ or presence $(+)$ of $1 \mu \mathrm{M}$ D600 for $48 \mathrm{~h}$. Cells were obtained from asthmatic (black columns; $n=5$ ), COPD (gray columns; $n=4$ ), and control subjects (white columns; $n=4$ ). Data are the mean \pm the SEM. ${ }^{*}, \mathrm{P}<0.05$ between populations within an experimental condition using ANOVA with the use of Bonferroni's test.,$+ \mathrm{P}<0.05$ between the absence and the presence of D600 within a population using paired Student's $t$ tests. 

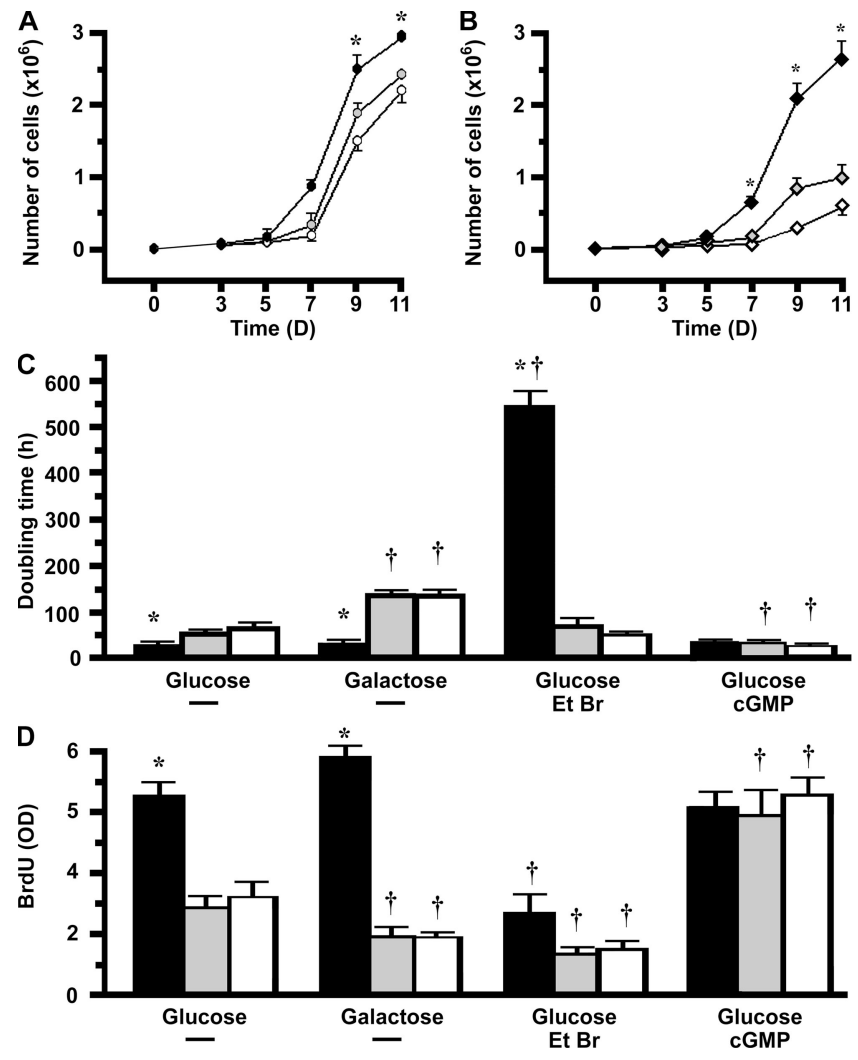

Figure 6. Asthmatic BSM cell proliferation is mitochondria dependent. BSM cell proliferation curves were obtained using either glucose (A) or galactose (B) in the culture medium. The doubling times of cell growth (C) were obtained from the proliferation curves. (D) BrdU incorporations were measured. BSM cells were cultured in various experimental conditions, i.e., glucose, galactose, glucose + ethidium bromide (Et Br), or glucose + cyclic GMP (cGMP). BSM cells were obtained from asthmatic (black symbols and columns; $n=4$ ), COPD (gray symbols and columns; $n=4$ ), and control subjects (white symbols and columns; $n=4$ ). Data are the mean \pm the SEM. ${ }^{*}, \mathrm{P}<0.05$ between populations within an experimental condition using ANOVA with the use of Bonferroni's test.,$+ \mathrm{P}<0.05$ between experimental conditions versus glucose within a population using paired Student's $t$ tests.

oxidative phosphorylations, whereas that of COPD and control subjects mainly uses mitochondrial-independent anaerobic glycolysis.

\section{Increased mitochondrial mass explains increased asthmatic BSM cell proliferation}

To determine whether the increased mitochondrial biogenesis found in asthmatic BSM cells is a cause or a consequence of the asthmatic BSM cell increased proliferation, three alternative approaches were used. First, mitochondria-deficient BSM cells were generated using culture with ethidium bromide, as previously described $(10,15)$. After $16 \mathrm{~d}$ of culture, all of the asthmatic BSM cells died, whereas those from both COPD patients and controls were still alive up to $30 \mathrm{~d}$ in the ethidium bromide medium (unpublished data). $6 \mathrm{~d}$ of incubation with ethidium bromide significantly decreased the amount of porin in the three groups of subjects (Fig. 7). Fig. $6 \mathrm{C}$ demonstrates that ethidium bromide significantly increased the doubling time of asthmatic BSM cells $(\mathrm{P}<0.001)$, but did not change that of both COPD $(\mathrm{P}=0.15)$ and control cells $(P=0.09)$. Similarly, proliferation of mitochondria-deficient asthmatic BSM cells decreased $(\mathrm{P}<0.001)$, whereas that of COPD and controls remained unchanged (Fig. S5, available at http://www.jem.org/cgi/content/full/jem.20070956/DC1). However, ethidium bromide also decreased BrdU incorporation of BSM cells from the three groups of subjects (Fig. $6 \mathrm{D}$ ). In a second alternative approach, we stimulated mitochondrial biogenesis using cyclic GMP for $6 \mathrm{~d}$, as previously described (16). The amount of mitochondria increased in such stimulated BSM cells from both COPD and controls (Fig. 7; $\mathrm{P}=0.02$ for both). Because the amount of mitochondria was already up-regulated in BSM cells from asthmatics, cyclic GMP failed to additionally increase the porin content $(\mathrm{P}=0.2)$. Cyclic GMP significantly decreased the doubling time $(\mathrm{P}=0.01)$, and increased the BrdU incorporation $(\mathrm{P}=0.02)$ and the proliferation of BSM cells from COPD patients $(\mathrm{P}=0.01)$ (Fig. 6, C and D, and Fig. S5). Similarly, cyclic GMP also decreased the doubling time $(\mathrm{P}<0.001)$ and increased the BrdU incorporation $(\mathrm{P}<0.001)$ and the proliferation of BSM cells from controls $(\mathrm{P}<0.001$; Fig. 6 , $\mathrm{C}$ and D, and Fig. S5). However, cyclic GMP had no effect in asthmatic BSM cell growth and proliferation. Finally, in a last approach, we analyzed the effect of altering calcium homeostasis on the proliferation of BSM cells. D600, which blocks calcium influx (Fig. $4 \mathrm{E}$ ) and the resulting increase in mitochondrial biogenesis and content only in asthmatic BSM cells (Fig. 5), also significantly inhibited the increased BrdU

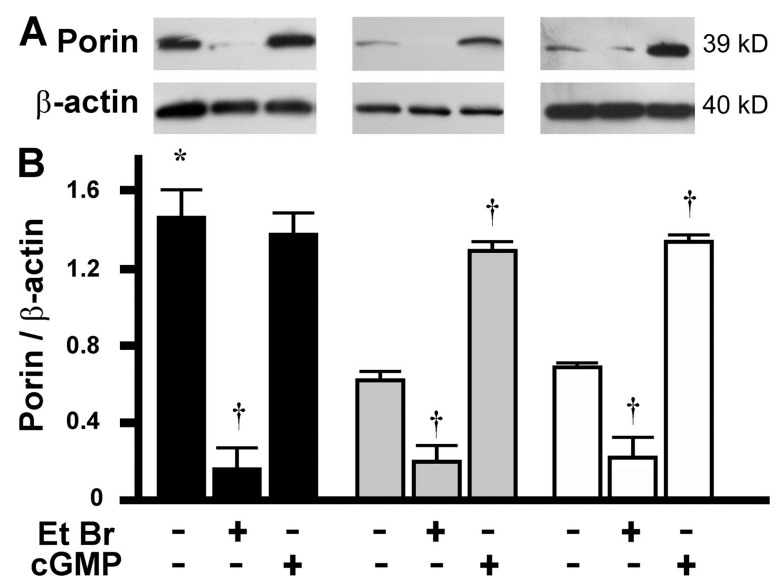

Figure 7. Effect of ethidium bromide and cyclic GMP on the porin content. Mitochondrial mass was assessed by the porin content using Western blot. BSM cells were obtained from asthmatic (black columns; $n=4$ ), COPD (gray columns; $n=4$ ), and control subjects (white columns; $n=4)$ and were cultured in the absence (-) or presence (+) of ethidium bromide (Et Br) or cyclic GMP (cGMP) for $6 \mathrm{~d}$ before the experiments. Data are the mean \pm the SEM. ${ }^{*}, \mathrm{P}<0.05$ between populations within an experimental condition using ANOVA with the use of Bonferroni's test. $t$, $\mathrm{P}<0.05$ between experimental conditions versus glucose within a population using paired Student's $t$ tests. 
incorporation in BSM cells from asthmatics, thus confirming that this enhanced cellular calcium influx represents the initial priming event (Fig. 8).

\section{DISCUSSION}

This study indicates that whereas both asthma and COPD are characterized by BSM remodeling, a specific mitochondria-dependent pathway is required for BSM proliferation only in asthma. This pathway is initiated by an altered calcium homeostasis, upon the activation of asthmatic BSM cells (Fig. S6, available at http://www.jem.org/cgi/content/full/ jem.20070956/DC1). Proliferation of BSM in both health and COPD is, at the very least, less mitochondria dependent. Thus, these results suggest that mitochondria may represent a specific new therapeutic target in airway remodeling in asthma.

In this study, we paid special attention when comparing data from severe asthmatics to that of COPD patients because both diseases have been shown to present smooth muscle remodeling $(2,3,17)$. Using a variety of different experimental approaches, we provide evidence that asthmatic BSM express a higher number of active mitochondria and a clear aspect of intense mitochondrial biogenesis. In our study, we assessed mitochondrial mass using various parameters, including the number of mitochondria by electron microscopy both ex vivo and in vitro, the mitochondrial network by confocal microscopy, and the porin content by Western blot. All of these methods provided consistent results. We also found significant correlations between in vitro BSM porin content, which is a relevant quantitative estimate of mitochondrial mass, and both the duration of the disease and the $\mathrm{FEV}_{1} / \mathrm{FVC}$ ratio within the asthmatic population. These correlations further support our hypothesis because, on the one hand, the longer the duration of the disease that is known to favor airway remodeling (18), the higher the mitochondrial mass, and on the other hand, the lower the $\mathrm{FEV}_{1} / \mathrm{FVC}$ ratio, which also reflects airway remodeling, the higher the mitochondrial mass. Moreover, to obtain a comprehensive assessment of mitochondrial content, we additionally measured the expression level of mtTFA by both

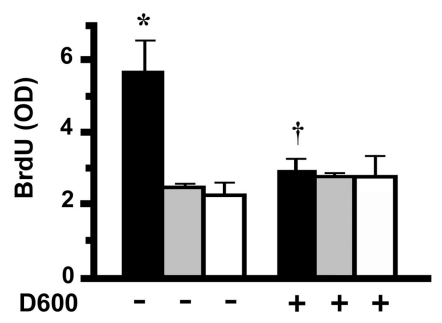

Figure 8. Effect of methoxyverapamil (D600) on BSM cell proliferation. BSM cell proliferation was measured using BrdU incorporations. Cells were cultured in the absence (-) or presence (+) of $1 \mu \mathrm{M}$ D600 for $48 \mathrm{~h}$. BSM cells were obtained from asthmatic (black columns; $n=4$ ), COPD (gray columns; $n=4$ ), and control subjects (white columns; $n=4$ ). Data are the mean \pm the SEM. ${ }^{*}, \mathrm{P}<0.05$ between populations within an experimental condition using ANOVA with the use of Bonferroni's test. $t$, $\mathrm{P}<0.05$ between the absence and the presence of $\mathrm{D} 600$ within a population using paired Student's $t$ tests. quantitative RT-PCR and Western blot to assess mitochondrial respiratory chain content, as mitochondrial overall content and respiratory chain content are not always linked (19).

The increased number of mitochondria was limited to asthmatic BSM cells compared with endothelial and epithelial cells from asthmatic, COPD, or control subjects. However, two characteristics of such asthmatic populations deserve further comment. First, it is unlikely that asthma treatments interfered with the observed changes because, on the one hand, 9/17 COPD patients took treatments similar to that of asthmatics, and, on the other hand, no difference has been found between controls and COPD. Second, the younger mean age of the asthmatic population is unlikely to contribute to this increase in mitochondrial content because (a) this increase persisted when comparing asthmatics to a subgroup of nonsmoking controls whose mean age was similar, and (b) in skeletal muscle, if not any other muscle, age does not influence mitochondrial content (20). To the best of our knowledge, this is the first description of such mitochondrial characteristics in any type of smooth muscle cells with potential pathophysiological implications. It has been previously reported that in a variety of differentiated tissues, a mitochondrial dysfunction increases mitochondrial biogenesis, suggesting a cellular compensatory mechanism (21). However, there was no mitochondrial dysfunction in our study because the respiration of asthmatic BSM mitochondria was efficiently coupled. Similarly, artificial activation of mitochondrial biogenesis of mouse neonatal cardiac myocytes largely induced a coupled respiration (22).

Mitochondrial biogenesis is controlled by many factors. PGC- $1 \alpha$ is a well-known master activator of mitochondrial biogenesis through the production of both NRF-1 and mtTFA in various cell types, including myoblast (23), fibroblast, or adipocytes (24). In this study, we demonstrated that such a cascade is activated in asthmatic BSM cells, as shown by the concomitant up-regulation of PGC-1 $\alpha, \mathrm{NRF}-1$, and mtTFA. Moreover, cyclic GMP has been shown to activate PGC-1 $\alpha$ and mitochondrial biogenesis in various cell lines, including U937, L6, and PC12 $(16,25)$. We have observed that cyclic GMP induces mitochondrial biogenesis in both control and COPD BSM cells, but not in asthmatic BSM cells. These findings suggest that mitochondrial biogenesis in asthmatic BSM cells may already be up-regulated. In this connection, cyclic GMP also improved cell proliferation of control and COPD BSM cells, but not those of asthmatics. It could be argued that cyclic GMP regulates many different genes in smooth muscle, such as vascular smooth muscle (26). However, cyclic GMP has been shown to inhibit, rather than enhance, the proliferation of vascular smooth muscle cells, as well as that of mesangial cells and various fibroblasts (26). In addition, direct improvement of mitochondrial biogenesis by transgenic overexpression of PGC-1 $\alpha$ activates skeletal muscle atrophy (27) and cardiac muscle dysfunction (22). Conversely, cyclic GMP increases the proliferation of endothelial cells, but the role of mitochondria in this phenomenon has not been investigated so far (28).

PGC- $1 \alpha$ activation can be calcium-dependent (29). In this study, we found that CaMK-IV was more phosphorylated in 
asthmatic BSM cells than in both controls and COPD. Rises in calcium concentration have been previously shown to activate CaMK-IV in other cell types, including skeletal muscle cells (29) or osteoclasts (30). Interestingly, we also demonstrated that calcium homeostasis in asthmatic BSM cells was altered, thus providing a mechanistic explanation for the increased activation of calcium-dependent signaling enzymes such as CaMK-IV (31). A D600-sensitive calcium influx accounts for such asthmainduced alteration in calcium homeostasis. We also provide evidence that this calcium influx was the initial priming event because, when blocked, mitochondrial biogenesis and subsequent asthmatic BSM cell-increased proliferation was inhibited. Whereas blockade of such influx may be beneficial, clinical responses remain to be examined because previous studies using methoxyverapamil were focused on short-term effects for up to $4 \mathrm{wk}$ and did not assess airway remodeling $(32,33)$.

Finally, we generated mitochondria-deficient BSM cells by depletion of mitochondrial DNA with ethidium bromide, which is a potent inhibitor of mitochondrial DNA replication and transcription (10,15). Mitochondria-deficient BSM cells from asthmatics were unable to proliferate, thereby confirming the importance of mitochondria in asthmatic BSM cell proliferation. Thus, the increased mitochondrial biogenesis observed in asthmatic BSM cells appears to be a cause rather than a consequence of the asthmatic BSM cell increased proliferation. However, it is well known that training can increase mitochondrial biogenesis in skeletal muscle (34). It is unlikely that a similar phenomenon appears to the BSM from asthmatics. In this study, none of the asthmatics presented recent exacerbations, and all of these patients were treated by relaxant $\beta 2$ agonists.

In conclusion, this study reveals that asthmatic BSM is characterized by an increased mitochondrial biogenesis that, in turn, enhances cell proliferation. Mitochondria may thus represent a new target for the treatment of asthmatic smooth muscle remodeling. Further studies are required to assess whether drugs interacting with mitochondrial biogenesis, including drugs acting at the site of calcium homeostasis, can prevent and/or reverse BSM remodeling in asthma.

\section{MATERIALS AND METHODS}

Study populations. A total of 14 patients with severe persistent asthma, 17 moderate to severe COPD patients, and 19 normal controls were prospectively recruited from the Centre Hospitalier Universitaire of Bordeaux according to both the Global Initiative for Asthma (35) and the Global Initiative for Chronic Obstructive Lung Disease guidelines (36). All subjects gave their written informed consent to participate in the study, after the nature of the procedure had been fully explained. The study followed recommendations outlined in the Helsinki Declaration and received approval from the local ethics committee. Bronchial specimens from all subjects were obtained by either fiberoptic bronchoscopy or lobectomy, as previously described $(37,38)$. A complete description of subjects is available in the Supplemental materials and methods (available at http://www.jem.org/cgi/content/full/jem.20070956/DC1).

Study procedures. Bronchial specimens were embedded in paraffin. Assessable BSM was identified by a pathologist in a blinded fashion using both morphological characteristics and $\alpha$-smooth muscle actin staining, as previously described (39). There was assessable BSM in the bronchial specimens from all 14 asthmatics, 17 COPD, and 19 controls. The total area of smooth muscle layer was calculated manually in a blinded fashion using ScanView software (Soft Imaging System) at a magnification of $200 \times$. This smooth muscle area was normalized by the whole area of the corresponding tissues and presented as percentages of whole area.

Bronchial specimens were also embedded in epon for electron microscopy (Tecnai 12; Philips), as previously described (39). 10 60-nm-thick ultrathin serial sections per specimen were cut and examined by a pathologist to locate whole nucleated BSM cells and endothelial and epithelial cells. The number of mitochondrial sections normalized by the whole-cell area and the density of mitochondria normalized by the cytoplasmic density were assessed manually in these 3 cell types from 10 to 25 measurements per section in a blinded fashion using ScanView and ImageJ (National Institutes of Health) softwares at a magnification of $6000 \times$.

Primary cultures of BSM cells were established from bronchial specimens, as previously described $(37,38,40)$. All experiments were performed on phenotypically confirmed smooth muscle cells between passages 2 and 4 . Cells were seeded on glass coverslips for confocal microscopy and microspectrofluorimetry, on culture flasks for electron microscopy and protein extraction, or on culture plates for RNA extraction. We used cells transfected with the plasmid mitochondrion-targeted GFP (mito-GFP) (14) to obtain confocal images of the mitochondrial network. Such images were acquired with FluoView laser scanning microscope (Nikon) and reconstituted in three-dimensional images using Imaris Software (Bitplane) (38). Immunoblotting was performed on cell protein extracts $(38,40)$ using primary antibodies directed against porin, mtTFA, NRF-1, and PGC-1 $\alpha$. After reverse transcription, real-time quantitative PCR was performed on a Rotor-Gene 2000 (Corbett Research) $(37,38,40)$, using appropriate primers designed to target mtTFA, NRF-1, or PGC-1 $\alpha$. Endogenous cell oxygen consumption, as well as coupling degree, were assessed in a thermostatically controlled chamber equipped with a Clark oxygen electrode (Oxygraph System; Hansatech), as previously described (14). Cell calcium was assessed by microspectrofluorimetry using Indo-1 probe, as previously described $(40,41)$. Cell proliferation was evaluated using both $\mathrm{BrdU}$ incorporation and cell counting. Cell apoptosis was finally studied using FITC-Annexin V and flow cytometry. A complete description of all methods is available in the Supplemental materials and methods.

Statistical analysis. The statistical analysis was performed with NCSS 2001 software. Comparison between the three groups was performed by means of ANOVA, with the use of Bonferroni's test for multiple comparisons or paired Student's $t$ tests. Values are presented as the mean \pm the SEM. A Pearson correlation matrix was built between in vitro and in vivo measurements. A P value $<0.05$ was considered statistically significant.

Online supplemental material. Fig. S1 provides ultrastructural characteristics of mitochondria from pellets of BSM cells. Fig. S2 demonstrates coupled endogenous BSM cell respiration. Fig. S3 provides BSM cell intracellular calcium responses to histamine. Fig. S4 shows spontaneous BSM cell apoptosis. Fig. S5 shows the effect of ethidium bromide and cyclic GMP on BSM cell proliferation. Fig. S6 illustrates the mechanisms of BSM proliferation in asthma. The online version of this article is available at http://www.jem .org/cgi/content/full/jem.20070956/DC1.

We would like to thank Liliane Dubuisson (SERCOMI, Université Bordeaux2) for technical assistance.

This study was supported by grants from the Fondation pour la Recherche Médicale, France (DAL 2005120574); the Agence Nationale de la Recherche, France (0591/ANR05 SEST 042-01); and the Programme Hospitalier de Recherche Clinique, Centre Hospitalier Universitaire de Bordeaux, France. D. Ghosh was funded by the Fondation pour la Recherche Médicale.

The authors have no conflicting financial interests.

Submitted: 11 May 2007

Accepted: 31 October 2007

\section{REFERENCES}

1. Busse, W.W., and R.F. Lemanske Jr. 2001. Asthma. N. Engl. J. Med. 344:350-362. 
2. Pepe, C., S. Foley, J. Shannon, C. Lemiere, R. Olivenstein, P. Ernst, M.S. Ludwig, J.G. Martin, and Q. Hamid. 2005. Differences in airway remodeling between subjects with severe and moderate asthma. J. Allergy Clin. Immunol. 116:544-549.

3. Hogg, J.C., F. Chu, S. Utokaparch, R. Woods, W.M. Elliott, L. Buzatu, R.M. Cherniack, R.M. Rogers, F.C. Sciurba, H.O. Coxson, and P.D. Pare. 2004. The nature of small-airway obstruction in chronic obstructive pulmonary disease. N. Engl. J. Med. 350:2645-2653.

4. Lambert, R.K., B.R. Wiggs, K. Kuwano, J.C. Hogg, and P.D. Pare 1993. Functional significance of increased airway smooth muscle in asthma and COPD. J. Appl. Physiol. 74:2771-2781.

5. Johnson, P.R., M. Roth, M. Tamm, M. Hughes, Q. Ge, G. King, J.K. Burgess, and J.L. Black. 2001. Airway smooth muscle cell proliferation is increased in asthma. Am. J. Respir. Crit. Care Med. 164:474-477.

6. Roth, M., P.R. Johnson, P. Borger, M.P. Bihl, J.J. Rudiger, G.G. King, Q. Ge, K. Hostettler, J.K. Burgess, J.L. Black, and M. Tamm. 2004. Dysfunctional interaction of C/EBPalpha and the glucocorticoid receptor in asthmatic bronchial smooth-muscle cells. N. Engl. J. Med. 351:560-574.

7. Ramos-Barbon, D., J.F. Presley, Q.A. Hamid, E.D. Fixman, and J.G Martin. 2005. Antigen-specific CD4+ T cells drive airway smooth muscle remodeling in experimental asthma. J. Clin. Invest. 115:1580-1589.

8. Jeffery, P.K. 2001. Remodeling in asthma and chronic obstructive lung disease. Am. J. Respir. Crit. Care Med. 164:S28-S38.

9. Springer, J., F.R. Scholz, C. Peiser, D.A. Groneberg, and A. Fischer. 2004. SMAD-signaling in chronic obstructive pulmonary disease: transcriptional down-regulation of inhibitory SMAD 6 and 7 by cigarette smoke. Biol. Chem. 385:649-653.

10. Holmuhamedov, E., L. Lewis, M. Bienengraeber, M. Holmuhamedova, A. Jahangir, and A. Terzic. 2002. Suppression of human tumor cell proliferation through mitochondrial targeting. FASEB J. 16:1010-1016.

11. Green, D.R., and G. Kroemer. 2004. The pathophysiology of mitochondrial cell death. Science. 305:626-629.

12. Oltersdorf, T., S.W. Elmore, A.R. Shoemaker, R.C. Armstrong, D.J. Augeri, B.A. Belli, M. Bruncko, T.L. Deckwerth, J. Dinges, P.J. Hajduk, et al. 2005. An inhibitor of Bcl-2 family proteins induces regression of solid tumours. Nature. 435:677-681.

13. Hackenbrock, C.R., T.G. Rehn, E.C. Weinbach, and J.J. Lemasters 1971. Oxidative phosphorylation and ultrastructural transformation in mitochondria in the intact ascites tumor cell. J. Cell Biol. 51:123-137.

14. Rossignol, R., R. Gilkerson, R. Aggeler, K. Yamagata, S.J. Remington, and R.A. Capaldi. 2004. Energy substrate modulates mitochondrial structure and oxidative capacity in cancer cells. Cancer Res. 64: 985-993.

15. King, M.P., and G. Attardi. 1989. Human cells lacking mtDNA: repopulation with exogenous mitochondria by complementation. Science. 246:500-503.

16. Nisoli, E., S. Falcone, C. Tonello, V. Cozzi, L. Palomba, M. Fiorani, A. Pisconti, S. Brunelli, A. Cardile, M. Francolini, et al. 2004. Mitochondrial biogenesis by $\mathrm{NO}$ yields functionally active mitochondria in mammals. Proc. Natl. Acad. Sci. USA. 101:16507-16512.

17. Ebina, M., T. Takahashi, T. Chiba, and M. Motomiya. 1993. Cellular hypertrophy and hyperplasia of airway smooth muscles underlying bronchial asthma. A 3-D morphometric study. Am. Rev. Respir. Dis. 148: $720-726$.

18. Bai, T.R., J. Cooper, T. Koelmeyer, P.D. Pare, and T.D. Weir. 2000. The effect of age and duration of disease on airway structure in fatal asthma. Am. J. Respir. Crit. Care Med. 162:663-669.

19. Benard, G., B. Faustin, E. Passerieux, A. Galinier, C. Rocher, N. Bellance, J.P. Delage, L. Casteilla, T. Letellier, and R. Rossignol. 2006. Physiological diversity of mitochondrial oxidative phosphorylation. Am. J. Physiol. Cell Physiol. 291:C1172-C1182.

20. Rasmussen, U.F., P. Krustrup, M. Kjaer, and H.N. Rasmussen. 2003. Experimental evidence against the mitochondrial theory of aging. A study of isolated human skeletal muscle mitochondria. Exp. Gerontol. 38:877-886

21. Hansson, A., N. Hance, E. Dufour, A. Rantanen, K. Hultenby, D.A. Clayton, R. Wibom, and N.G. Larsson. 2004. A switch in metabolism precedes increased mitochondrial biogenesis in respiratory chain-deficient mouse hearts. Proc. Natl. Acad. Sci. USA. 101:3136-3141.
22. Lehman, J.J., P.M. Barger, A. Kovacs, J.E. Saffitz, D.M. Medeiros, and D.P. Kelly. 2000. Peroxisome proliferator-activated receptor gamma coactivator1 promotes cardiac mitochondrial biogenesis. J. Clin. Invest. 106:847-856.

23. Wu, Z., P. Puigserver, U. Andersson, C. Zhang, G. Adelmant, V. Mootha, A. Troy, S. Cinti, B. Lowell, R.C. Scarpulla, and B.M. Spiegelman. 1999 Mechanisms controlling mitochondrial biogenesis and respiration through the thermogenic coactivator PGC-1. Cell. 98:115-124.

24. Gleyzer, N., K. Vercauteren, and R.C. Scarpulla. 2005. Control of mitochondrial transcription specificity factors (TFB1M and TFB2M) by nuclear respiratory factors (NRF-1 and NRF-2) and PGC-1 family coactivators. Mol. Cell. Biol. 25:1354-1366.

25. Nisoli, E., E. Clementi, C. Paolucci, V. Cozzi, C. Tonello, C. Sciorati, R. Bracale, A. Valerio, M. Francolini, S. Moncada, and M.O. Carruba. 2003. Mitochondrial biogenesis in mammals: the role of endogenous nitric oxide. Science. 299:896-899.

26. Pilz, R.B., and D.E. Casteel. 2003. Regulation of gene expression by cyclic GMP. Circ. Res. 93:1034-1046.

27. Miura, S., E. Tomitsuka, Y. Kamei, T. Yamazaki, Y. Kai, M. Tamura, K. Kita, I. Nishino, and O. Ezaki. 2006. Overexpression of peroxisome proliferator-activated receptor gamma co-activator-1alpha leads to muscle atrophy with depletion of ATP. Am. J. Pathol. 169:1129-1139.

28. Parenti, A., L. Morbidelli, X.L. Cui, J.G. Douglas, J.D. Hood, H.J. Granger, F. Ledda, and M. Ziche. 1998. Nitric oxide is an upstream signal of vascular endothelial growth factor-induced extracellular signalregulated kinase1/2 activation in postcapillary endothelium. J. Biol. Chem. 273:4220-4226

29. Wu, H., S.B. Kanatous, F.A. Thurmond, T. Gallardo, E. Isotani, R. Bassel-Duby, and R.S. Williams. 2002. Regulation of mitochondrial biogenesis in skeletal muscle by CaMK. Science. 296:349-352.

30. Sato, K., A. Suematsu, T. Nakashima, S. Takemoto-Kimura, K. Aoki, Y. Morishita, H. Asahara, K. Ohya, A. Yamaguchi, T. Takai, et al. 2006. Regulation of osteoclast differentiation and function by the CaMK-CREB pathway. Nat. Med. 12:1410-1416.

31. Bootman, M.D., and M.J. Berridge. 1995. The elemental principles of calcium signaling. Cell. 83:675-678.

32. Massey, K.L., M. Hill, E. Harman, D.R. Rutledge, R. Ahrens, and L. Hendeles. 1988. Dose response of inhaled gallopamil (D600), a calcium channel blocker, in attenuating airway reactivity to methacholine and exercise. J. Allergy Clin. Immunol. 81:912-918.

33. Ahmed, T., C.S. Kim, and I. Danta. 1988. Inhibition of antigen-induced bronchoconstriction by a new calcium antagonist, gallopamil: comparison with cromolyn sodium. J. Allergy Clin. Immunol. 81:852-858.

34. Hood, D.A. 2001. Invited Review: contractile activity-induced mitochondrial biogenesis in skeletal muscle. J. Appl. Physiol. 90:1137-1157.

35. Global Initiative for Asthma. 1995. Global strategy for asthma management and prevention. NIH publication (updated 2005). http://www .ginasthma.com/download.asp?intId=187

36. Global Initiative for Chronic Obstructive Lung Disease. 1998. Global strategy for the diagnosis, management and prevention of chronic obstructive pulmonary disease. NIH publication (updated 2005). http:// www.goldcopd.com/download.asp?intId=231

37. Berger, P., P.O. Girodet, H. Begueret, O. Ousova, D.W. Perng, R. Marthan, A.F. Walls, and J.M. Tunon De Lara. 2003. Tryptase-stimulated human airway smooth muscle cells induce cytokine synthesis and mast cell chemotaxis. FASEB J. 17:2139-2141.

38. El-Shazly, A., P. Berger, P.O. Girodet, O. Ousova, M. Fayon, J.M Vernejoux, R. Marthan, and J.M. Tunon-de-Lara. 2006. Fraktalkine produced by airway smooth muscle cells contributes to mast cell recruitment in asthma. J. Immunol. 176:1860-1868.

39. Begueret, H., P. Berger, J.M. Vernejoux, L. Dubuisson, R. Marthan, and J.M. Tunon-de-Lara. 2007. Inflammation of bronchial smooth muscle in allergic asthma. Thorax. 62:8-15.

40. Trian, T., P.O. Girodet, O. Ousova, R. Marthan, J.M. Tunon-de-Lara, and P. Berger. 2006. RNA interference decreases PAR-2 expression and function in human airway smooth muscle cells. Am. J. Respir. Cell Mol. Biol. 34:49-55.

41. Berger, P., J.M. Tunon-de-Lara, J.P. Savineau, and R. Marthan. 2001. Tryptase-induced $\mathrm{PAR}-2$-mediated $\mathrm{Ca}(2+)$ signaling in human airway smooth muscle cells. J. Appl. Physiol. 91:995-1003. 